\title{
The Impact of Aorto-mitral Angle on New Onset Atrial Arrhythmia after Mitral Valve Repair in Patients with Mitral Regurgitation
}

\author{
Shunsuke Kagawa ${ }^{1}$, Yukio Abe ${ }^{1}$, Yoshiki Matsumura ${ }^{1}$, Nanaka Nomura ${ }^{1}$, Kenji Shimeno ${ }^{1}$, \\ Takahiko Naruko ${ }^{1}$, Takashi Murakami ${ }^{1}$, Yosuke Takahashi ${ }^{2}$, Toshihiko Shibata ${ }^{2}$, and \\ Minoru Yoshiyama \\ ${ }^{1}$ Osaka City General Hospital \\ ${ }^{2}$ Osaka City University
}

February 13, 2021

\begin{abstract}
Background: We hypothesized that the post-operative aorto-mitral angle might relate to the occurrence of post-operative atrial arrhythmia (AA), including atrial fibrillation and atrial tachycardia, after mitral valve repair in patients with mitral regurgitation (MR). The purpose of the present study was to determine the effects of the post-operative aorto-mitral angle on new onset AA after mitral valve repair with mitral annuloplasty for treating MR. Methods: One-hundred seventy-two patients without any history of AA underwent mitral valve repair with mitral annuloplasty in our institution between 2008 and 2017. Patient information, including medical records and echocardiographic data, were retrospectively studied. Results: AA occurred in 15 (8.7\%) patients during the follow-up period (median, 35.7 months; range, 0.5-132 months). The patients with AA had a longer cardiopulmonary bypass time and a smaller aorto-mitral angle at post-operative TTE than the others (119 \pm 6 degrees vs. $125 \pm 10$ degrees, $\mathrm{P}=0.003)$. There was no significant difference in the degree of post-operative residual MR or functional MS between the groups. In a multivariate Cox proportional hazards analysis, the longer cardiopulmonary bypass time and the smaller post-operative aorto-mitral angle were independent predictors of the occurrence of AA during the follow-up period (odds ratio per 10 minutes $1.11 ; 95 \%$ CI 1.02-1.22, $\mathrm{P}=0.019$ : odds ratio 0.91; 95\% CI 0.85-0.98, $\mathrm{P}=0.012$ ). Conclusion: A small aorto-mitral angle at post-operative TTE was a predictor of new onset AA after a mitral valve repair for treating MR.
\end{abstract}

The Impact of Aorto-mitral Angle on New Onset Atrial Arrhythmia after

Mitral Valve Repair in Patients with Mitral Regurgitation

Shunsuke Kagawa (MD) ${ }^{1}$, Yukio Abe $(\mathrm{MD}, \mathrm{PhD})^{1}$,

Yoshiki Matsumura (MD, PhD) ${ }^{1}$, Nanaka Nomura (MD) ${ }^{1}$,

Kenji Shimeno (MD, PhD) ${ }^{1}$, Takahiko Naruko (MD, PhD $)^{1}$,

Takashi Murakami (MD, PhD) ${ }^{2}$, Yosuke Takahashi (MD, PhD) ${ }^{3}$,

Toshihiko Shibata (MD, PhD) ${ }^{3}$, Minoru Yoshiyama (MD, PhD $)^{4}$

${ }^{1}$ Department of Cardiology, Osaka City General Hospital, Osaka, Japan

${ }^{2}$ Department of Cardiovascular Surgery, Osaka City General Hospital, Osaka, Japan

${ }^{3}$ Department of Cardiovascular Surgery, Osaka City University, Osaka, Japan

${ }^{4}$ Department of Cardiology, Osaka City University, Osaka, Japan 
Word count: structured abstract, 240 words; text, authors' contributions, references and figure legends, 2707 words.

\section{Corresponding author:}

Yukio Abe, MD, PhD, Department of Cardiology, Osaka City General Hospital,

2-13-22 Miyakojima-hondori, Miyakojima-ku, Osaka, Japan 534-0021

E-mail: abeyukio@aol.com Phone: (+81)-6-6929-1221 Fax: (81)-6-6929-1090

\section{Abstract}

Background: We hypothesized that the post-operative aorto-mitral angle might relate to the occurrence of post-operative atrial arrhythmia (AA), including atrial fibrillation and atrial tachycardia, after mitral valve repair in patients with mitral regurgitation (MR). The purpose of the present study was to determine the effects of the post-operative aorto-mitral angle on new onset AA after mitral valve repair with mitral annuloplasty for treating MR.

Methods: One-hundred seventy-two patients without any history of AA underwent mitral valve repair with mitral annuloplasty in our institution between 2008 and 2017. Patient information, including medical records and echocardiographic data, were retrospectively studied.

Results: AA occurred in 15 (8.7\%) patients during the follow-up period (median, 35.7 months; range, 0.5-132 months). The patients with AA had a longer cardiopulmonary bypass time and a smaller aorto-mitral angle at post-operative TTE than the others $(119 \pm 6$ degrees vs. $125 \pm 10$ degrees, $\mathrm{P}=0.003)$. There was no significant difference in the degree of post-operative residual MR or functional MS between the groups. In a multivariate Cox proportional hazards analysis, the longer cardiopulmonary bypass time and the smaller post-operative aorto-mitral angle were independent predictors of the occurrence of AA during the follow-up period (odds ratio per 10 minutes $1.11 ; 95 \%$ CI 1.02-1.22, $\mathrm{P}=0.019$ : odds ratio $0.91 ; 95 \%$ CI $0.85-0.98, \mathrm{P}$ $=0.012$ ).

Conclusion: A small aorto-mitral angle at post-operative TTE was a predictor of new onset AA after a mitral valve repair for treating MR.

\section{Keywords}

Aorto-mitral angle; Atrial arrhythmia; Echocardiography; Mitral regurgitation; Mitral valve repair

\section{Abbreviations:}

$\mathrm{AA}=$ atrial arrhythmia

$\mathrm{AF}=$ atrial fibrillation

$\mathrm{AT}=$ atrial tachycardia

$\mathrm{CPB}=$ cardiopulmonary bypass

$\mathrm{EF}=$ ejection fraction

$\mathrm{LA}=$ left atrium or left atrial

$\mathrm{LV}=$ left ventricle or left ventricular

$\mathrm{MR}=$ mitral regurgitation

$\mathrm{ROC}=$ receiver operating characteristic

$\mathrm{TMF}=$ transmitral flow

$\mathrm{TMPG}=$ transmitral pressure gradient 
TTE $=$ transthoracic echocardiography

\section{INTRODUCTION}

New onset atrial arrhythmia (AA), including atrial fibrillation (AF) and atrial tachycardia (AT), can occur after mitral valve repair in patients with mitral regurgitation (MR). Post-operative AF has been reported to be associated with long-term all-cause mortality. ${ }^{1,2}$ However, a predictor of post-operative AA has not been fully elucidated. A previous study has shown that the post-operative aorto-mitral angle is associated with the reverse remodeling of the left atrium (LA) after a mitral valve repair. ${ }^{3}$ Consequently, we hypothesized that the post-operative aorto-mitral angle might also relate to the occurrence of post-operative AA. The purpose of the present study was to determine whether the post-operative aorto-mitral angle could predict new onset AA after mitral valve repair with mitral annuloplasty for treating MR.

\section{METHODS}

\subsection{Patients and study protocol}

We retrospectively studied 268 consecutive patients who underwent a primary mitral valve repair for treating MR from January 2008 to December 2017 in Osaka City General Hospital, Osaka, Japan. We excluded patients: 1) who were treated without mitral annuloplasty $(\mathrm{n}=10), 2)$ who underwent intraoperative conversion to mitral valve replacement $(\mathrm{n}=3)$ and 3$)$ who had a history of paroxysmal or chronic AF/AT $(\mathrm{n}=83)$. Consequently, we enrolled 172 patients as a final cohort in this retrospective study. The clinical, echocardiographic and follow-up data were collected from the medical records and the echocardiographic database. The end-diastolic aorto-mitral angle was additionally measured from the digital echocardiographic images at the pre-operative and post-operative periods. Patients were generally followed up at our outpatient clinics 3, 6 and 12 months after their surgeries and yearly thereafter. The post-operative AA event was defined as paroxysmal or persistent AF or AT that occurred after the hospital discharge, irrespective of the presence or absence of symptoms. AA events that occurred before discharge were not considered post-operative AA events. The protocol of this retrospective study was approved by the institutional review board of the Osaka City General Hospital, with a waiver of individual consent.

\subsection{Echocardiographic studies}

Transthoracic echocardiography (TTE) was routinely performed before and 7-14 days after mitral valve repair. Left ventricular (LV) diastolic dimension, LV systolic dimension, indexed LV mass, LV ejection fraction (EF), LA dimension, the severity of MR (none, mild, moderate or severe), mean transmitral pressure gradient (TMPG), the severity of tricuspid regurgitation (none, mild, moderate or severe) and peak systolic transtricuspid pressure gradient were evaluated according to the guidelines. ${ }^{4-7}$ Post-operative functional MS was considered significant when a mean TMPG was [?] $5 \mathrm{mmHg}$, despite the absence of apparent sclerosis of the leaflets or a moderate or more degree of residual MR. The aorto-mitral angle was measured from the recorded digital images as the intersection angle of the mitral annulus ring and aortic valve plane at end-diastolic on parasternal long axis images, as shown in Figure $1 .^{3}$

\subsection{Surgical procedure}

The technical details of the mitral valve repair procedure were determined at the surgeon's discretion according to the mitral morphology. There were two approaches for the mitral valve: an incision to the LA and an incision to the right atrium and atrial septum. The techniques for leaflet repair were selected from a new chordal replacement, leaflet resection (quadrangular or triangular leaflet resection) and leaflet augmentation. A semi-rigid full ring or a flexible band was implanted in all patients.

\subsection{Statistical analysis}

The categorical variables are expressed as absolute values and percentages and were compared using a chisquared test. The continuous variables are expressed as mean +- SD and were compared using a t-test. Univariate and multivariate Cox proportional hazards analysis were applied to study the determinants of 
post-operative AA events among the parameters of clinical, echocardiographic and surgical data. Multivariate analyses were performed using stepwise selection with models using significant factors from univariate analyses. Sensitivities, specificities, positive predictive values, negative predictive values and accuracies for predicting AA events were calculated using standard methods. Receiver operating characteristic (ROC) curves and the areas under the ROC curves were assessed using standard methods. The optimal cut-off value was defined as that with highest sum of sensitivity and specificity. Survival curves were produced according to the Kaplan-Meier method, and differences between groups were compared with the log-rank test. A P-value $<0.05$ was considered statistically significant. All statistical analyses were performed with EZR (Saitama Medical Center, Jichi Medical University, Saitama, Japan), which is a graphical user interface for $\mathrm{R}$ (The R Foundation for Statistical Computing, Vienna, Austria). More precisely, it is a modified version of $\mathrm{R}$ commander designed to add statistical functions frequently used in biostatistics.

\section{RESULTS}

The baseline characteristics and pre-operative TTE data, including MR etiologies, are listed in Table 1. The mean age was $62+-13$ years, and $105(61 \%)$ patients were men. The mean value of the LV ejection fraction was $64.6+-8.7 \%$. A large proportion $(87 \%)$ had a prolapse of the anterior and/or posterior mitral leaflets due to fibroelastic deficiency as the main etiology of MR. Fifteen patients experienced new onset AA events, including paroxysmal or persistent AF and AT during the post-operative follow-up period, with a median of 35.7 months (range, 0.5 to 132 months). No significant difference was found in baseline characteristics between patients who experienced post-operative AA events and those who didn't. Table 2 shows the data for the surgical procedure. There were no significant differences in any pre-operative TTE parameter between the two groups, including the MR etiology, extent of mitral valve lesions and aorto-mitral angle (135 +- 11 degrees vs. $131+-12$ degrees, $\mathrm{P}=0.29)$. The cardiopulmonary bypass $(\mathrm{CPB})$ time was longer in patients who had post-operative AA events than in patients who didn't $(195+-69$ min vs. $168+-53$ min, $\mathrm{P}=$ 0.035). The post-operative TTE data are listed in Table 3. The patients who experienced post-operative AA events had a smaller aorto-mitral angle at the post-operative TTE than the patients who didn't (119 +6 degrees vs. $125+-10$ degrees, $\mathrm{P}=0.003$ ). There was no significant difference in the grading of residual MR or the mean TMPG between the two groups.

The univariate Cox proportional hazards analyses revealed that the CPB time and the aorto-mitral angle at the post-operative TTE were significant predictors of the post-operative AA events among all demographic, procedural and echocardiographic parameters. A multivariate Cox proportional hazards analysis revealed that the CPB time (odds ratio 1.12 per 10 minutes; $95 \%$ CI 1.03-1.21; $\mathrm{P}=0.008$ ) and the aorto-mitral angle at the post-operative TTE (odds ratio $0.92 ; 95 \%$ CI $0.86-0.98 ; \mathrm{P}=0.014$ ) were the independent predictors of the new onset AA events (Table 4). The area under the ROC curve of the aorto-mitral angle to predict the new onset AA event was 0.717 (Figure 2). One-hundred and seventeen degrees was the optimal cutoff value of the post-operative aorto-mitral angle for predicting the post-operative AA events with a sensitivity of $57 \%$ (95\% CI, 29-82\%) and a specificity of $80 \%$ (95\% CI, 73-86\%). Figure 3 shows the Kaplan-Meier event-free curves in patients stratified based on the post-operative aorto-mitral angle. The post-operative AA event-free rate was higher in patients with the post-operative aorto-mitral angle $>117$ degrees than in patients with the post-operative aorto-mitral angle [?] 117 degrees.

We additionally analyzed the determinants of the small post-operative aorto-mitral angle ([?] 117 degrees). TMPG $>3 \mathrm{mmHg}$ (odds ratio 2.53; 95\% CI, 1.19-5.37, $\mathrm{P}=0.016$ ) was the only determinant of the small post-operative aorto-mitral angle among the post-operative echocardiographic parameters.

\section{DISCUSSION}

In the present study, we examined the predictors of post-operative AA, including AF and AT occurring after mitral valve repair for treating MR. We found that: 1) $9 \%$ of patients who underwent mitral valve repair for treating $\mathrm{MR}$ met $\mathrm{AA}$ in the post-operative follow-up period, 2) the aorto-mitral angle and the CPB time were independent predictors of the post-operative AA and 3) the patients with post-operative aorto-mitral angle

[?] 117 degrees had a higher rate of post-operative AA than patients with the post-operative aorto-mitral 
angle $>117$ degrees.

New onset AA, including AF and AT, is the common arrhythmia after mitral valve repair in patients with $\mathrm{MR}$, and peri-operative and post-operative AF have been reported to be associated with long-term all-cause mortality. ${ }^{1,8}$ Therefore, the prediction and prevention of AA after mitral valve repair in patients of MR can contribute to improving their post-operative prognosis. Our results revealed that the aorto-mitral angle is an independent predictor of post-operative AA. A previous report indicated that a small aorto-mitral angle after mitral valve repair for MR was an independent predictor of systolic anterior motion leading to left ventricular outflow obstruction. ${ }^{9}$ However, there are very few reports about the relationship between the aorto-mitral angle and AA we present in this study. A previous study has shown that post-operative aorto-mitral angle is associated with the reduction of the LA diameter after mitral valve repair, and LA enlargement is an important risk factor for the incidence of late AF. ${ }^{3,10,11}$ The small aorto-mitral angle represents the tilting of the mitral annular plane and may functionally narrow the diastolic mitral orifice (Figure 4), which is considered to act on a similar principle to that of functional mitral stenosis after mitral valve repair for ischemic functional MR. ${ }^{12,13}$

In the present study, high TMPG ( $>3 \mathrm{mmHg}$ ) was independently associated with small aorto-mitral angle. Consequently, we hypothesized that a small aorto-mitral angle functionally narrowed the diastolic mitral orifice and increased the TMPG, resulting in the progression of LA dilatation and the occurrence of the AA. However, neither post-operative mean TMPG nor LA diameter was directly associated with an AA event in our present results. Exercise stress tests can diagnose patients with subclinical functional MS after mitral valve repair by determining the effective orifice area index and pulmonary arterial pressure that are associated with exercise capacity. ${ }^{13,14}$ Otherwise, assessments of follow-up echocardiographic data may be required to prove the association among the aorto-mitral angle, TMPG, LA size and AA events. Further studies will be needed to address this issue.

Various studies have reported that mitral valve repair with ring annuloplasty affects the angle between the planes of the mitral valve and the aortic valve. Some previous studies have reported that the implantation of an undersized ring was associated with a small aorto-mitral angle and a great TMPG. ${ }^{15,16}$ Avoidance of the selection of an undersized ring may be useful in preventing a small aorto-mitral angle leading to the occurrence of post-operative AA events. A previous study has shown that mitral annuloplasty with a C-shape band leads to a smaller aorto-mitral angle than those with a full ring. ${ }^{3}$ The selection of the appropriate size and type of an artificial ring can prevent a constrictive aorto-mitral angle and the occurrence of AA events. Another previous study reported that mitral valve repair with LA plication can also prevent the narrowing of the post-operative mitral-septal angle, which is a parameter possessing a pathological significance similar to that of the aorto-mitral angle. ${ }^{17}$

\subsection{Limitations}

There are several limitations in our study. The main limitation of the present study was its retrospective observational protocol. Second, the surgeons were not constant through the series, and third, the AA events were confirmed only when objectively documented. The occurrence of asymptomatic transient AF might not be detected, and the incidence of AA might be underestimated. Finally, the sample size was relatively small, and the statistical power of the analyses was not strong. Further studies are needed to definitively determine if the small aorto-mitral angle leads to the AA events.

\section{CONCLUSION}

A small aorto-mitral angle at post-operative TTE affects new onset AA in patients who underwent mitral valve repair for treating MR. Consequently, the probability of post-operative AA may decrease with surgical procedures to prevent the narrowing of the aorto-mitral angle large.

\section{AUTHORS CONTRIBUTIONS}

Shunsuke Kagawa: principal investigator, data analysis, manuscript writing 
Yukio Abe: echocardiographic measurements, critical review of manuscript

Nanaka Nomura: data collections and echocardiographic measurements

Shimeno Kenji: data collections and echocardiographic measurements

Yoshiki Matsumura: data collections and echocardiographic measurements

Takashi Murakami: attending surgeons who performed the mitral valve surgeries

Yosuke Takahashi: attending surgeons who performed the mitral valve surgeries

Toshihiko Shibata: attending surgeons who performed the mitral valve surgeries

Minoru Yoshiyama: overall supervision and critical review of manuscript

\section{REFERENCES}

1. Bramer S, van Straten AH, Soliman Hamad MA, et al. New-onset postoperative atrial fibrillation predicts late mortality after mitral valve surgery. Ann Thorac Surg. 2011;92(6):2091-2096

2. Villareal RP, Hariharan R, Liu BC, et al. Postoperative atrial fibrillation and mortality after coronary artery bypass surgery. J Am Coll Cardiol. 2004;43(5):742-748.

3. Ma W, Ye W, Zhang J, et al. Impact of different annuloplasty rings on geometry of the mitral annulus with fibroelastic deficiency: the significance of aorto-mitral angle. Int J Cardiovasc.2018;34(11):1707-1713.

4. Lang RM, Bierig M, Devereux RB, et al. Chamber Quantification Writing Group, American Society of Echocardiography's Guidelines and Standards Committee, \& European Association of Echocardiography, Recommendations for chamber quantification: a report from the American Society of Echocardiography's Guidelines and Standards Committee and the Chamber Quantification Writing Group, developed in conjunction with the European Association of Echocardiography, a branch of the European Society of Cardiology. $J$ Am Soc Echocardiogr. 2005;18(2):1440-1463.

5. Rudski LG, Lai WW, Afilalo J, et al. Guidelines for the echocardiographic assessment of the right heart in adults: a report from the American Society of Echocardiography endorsed by the European Association of Echocardiography, a registered branch of the European Society of Cardiology, and the Canadian Society of Echocardiography. J Am Soc Echocardiogr. 2010;23(7):685-788.

6. Zoghbi WA, Enriquez-Sarano M, Foster E, et al. Recommendations for evaluation of the severity of native valvular regurgitation with two-dimensional and Doppler echocardiography. J Am Soc Echocardiogr. 2003;16(7):777-802.

7. Quinones MA, Otto CM, Stoddard M, et al. Recommendations for quantification of Doppler echocardiography: a report from the Doppler Quantification Task Force of the Nomenclature and Standards Committee of the American Society of Echocardiography. J Am Soc Echocardiogr.2002;15(2):167-184.

8. Melduni RM, Schaff HV, Bailey KR, et al. Implications of new-onset atrial fibrillation after cardiac surgery on long-term prognosis: a community-based study. Am Heart J. 2015;170(4):659-668.

9. Varghese R, Itagaki S, Anyanwu AC, et al. Predicting systolic anterior motion after mitral valve reconstruction: using intraoperative transesophageal echocardiography to identify those at greatest risk. Eur $J$ Cardiothorac Surg. 2014;45(1): 132-138.

10. Kernis SJ, Nkomo VT, Messika-Zeitoun D, et al. Atrial fibrillation after surgical correction of mitral regurgitation in sinus rhythm: incidence, outcome, and determinants. Circulation. 2004;110(16): 2320-2325.

11. Magruder JT, Collica S, Belmustakov S, et al. Predictors of late-onset atrial fibrillation following isolated mitral valve repairs in patients with preserved ejection fraction. J Card Surg.2016;31(8):486-492. 
12. Kubota K, Otsuji Y, Ueno T, et al. Functional mitral stenosis after surgical annuloplasty for ischemic mitral regurgitation: importance of subvalvular tethering in the mechanism and dynamic deterioration during exertion. J Thorac Cardiovasc Surg. 2010;140(3): 617-623.

13. Bertrand PB, Verbrugge FH, Verhaert D, et al. Mitral valve area during exercise after restrictive mitral valve annuloplasty: importance of diastolic anterior leaflet tethering. $J$ Am Coll Cardiol. 2015;65 (5):452-461.

14. Samiei N, Tajmirriahi M, Rafati A, et al. Pulmonary arterial pressure detects functional mitral stenosis after annuloplasty for primary mitral regurgitation: An exercise stress echocardiographic study. Echocardiogra. 2018;35(2): 211-217.

15. Witschey WR, Zhang D, Contijoch F, et al. The influence of mitral annuloplasty on left ventricular flow dynamics. Ann Thorac Surg.2015;100(1):114-121.

16. Kawamoto N, Fujita T, Hata H, et al. Prosthesis-patient mismatch due to small ring annuloplasty in patients with degenerative mitral insufficiency. J Cardiol. 2016;68(2):141-147.

17. Kaneyuki D, Nakajima H, Asakura T, et al. The change in the mitral-septal angle after surgery for atrial functional mitral regurgitation. Gen Thorac Cardiovasc Surg. 2021;69(1):1-7.

\section{Figure legends}

Figure 1. Measurement of the aorto-mitral angle in transthoracic echocardiography.

Figure 2. The receiver operating characteristic curve of postoperative aorto-mitral angle to predict new onset atrial arrhythmia.

Figure 3. The Kaplan-Meier curve estimating the freedom from new onset atrial arrhythmia in the cohort.

Figure 4. An example of a post-operative aorto-mitral angle narrowed after surgery. The dashed arrow indicates the direction of the transmitral flow (TMF) during diastole. The small aorto-mitral angle represents the tilting of the mitral annular plane and the angle between the direction of the TMF, sharpening the mitral annular plane. Consequently, the diastolic mitral orifice is functionally narrowed.

Table 1. Baseline patients' characteristics and pre-operative echocardiography.

\begin{tabular}{lllll}
\hline & All $(\mathrm{n}=172)$ & No event group $(\mathrm{n}=157)$ & AA event group $(\mathrm{n}=15)$ & $\mathrm{P}$ \\
\hline Age, year & $61.6 \pm 13.3$ & $61.4 \pm 13.5$ & $63.7 \pm 11.0$ & 0.52 \\
Male, n (\%) & $105(61)$ & $94(59)$ & $11(73)$ & 0.41 \\
Body surface area, m ${ }^{2}$ & $1.62 \pm 0.22$ & $1.61 \pm 0.22$ & $1.64 \pm 0.22$ & 0.59 \\
Hypertension, n (\%) & $85(49)$ & $77(49)$ & $8(53)$ & 0.96 \\
Diabetes mellitus, $\mathrm{n}(\%)$ & $18(10)$ & $15(10)$ & $3(20)$ & 0.19 \\
Hypercholesterolemia, n (\%) & $52(30)$ & $48(31)$ & $4(27)$ & 0.98 \\
Smoking, n (\%) & $51(30)$ & $46(29)$ & $4(27)$ & 0.98 \\
Coronary artery disease, n (\%) & $23(13)$ & $19(12)$ & $0(0)$ & 0.24 \\
Chronic kidney disease, n (\%) & $14(8)$ & $14(9)$ & 2 & 0.48 \\
NYHA & & & 9 & \\
I & 47 & 45 & 4 & \\
II & 96 & 87 & 0 & \\
III & 24 & 20 & $121 \pm 28$ & 0.32 \\
IV & 5 & 5 & $51.9 \pm 5.4$ & 0.36 \\
Echocardiographic data & $116 \pm 32$ & $115 \pm 32$ & $32.2 \pm 3.7$ & 0.42 \\
LV mass index, g/m & & & \\
LV end-diastolic diameter, mm & $53.3 \pm 6.1$ & $53.4 \pm 6.2$ & \\
LV end-systolic diameter, mm & $33.4 \pm 6.1$ & $33.5 \pm 6.3$ & &
\end{tabular}




\begin{tabular}{lllll}
\hline & All $(\mathrm{n}=172)$ & No event group $(\mathrm{n}=157)$ & AA event group $(\mathrm{n}=15)$ & $\mathrm{P}$ \\
\hline LV ejection fraction, $\%$ & $64.6 \pm 8.7$ & $64.4 \pm 9.0$ & $66.4 \pm 5.4$ & 0.42 \\
LA diameter, mm & $41.4 \pm 6.7$ & $41.5 \pm 6.7$ & $41.9 \pm 6.8$ & 0.85 \\
TR peak pressure gradient, mmHg & $32.6 \pm 15.5$ & $32.3 \pm 14.9$ & $35.0 \pm 20.0$ & 0.59 \\
Aorto-mitral angle, degree & $134 \pm 11$ & $135 \pm 11$ & $131 \pm 12$ & 0.29 \\
Etiology of MR & & & & \\
Prolapse due to FED, n (\%) & $150(87)$ & $135(86)$ & $15(100)$ & 0.61 \\
Anterior mitral leaflet prolapse, n (\%) & $59(34)$ & $50(32)$ & $9(60)$ & 0.051 \\
Posterior mitral leaflet prolapse, n (\%) & $111(65)$ & $104(66)$ & $7(47)$ & 0.08 \\
Barlow's disease, n (\%) & $3(2)$ & $3(2)$ & $0(0)$ & 1 \\
Rheumatic, n (\%) & $1(1)$ & $1(1)$ & $0(0)$ & 1 \\
Secondary MR, n (\%) & $4(2)$ & $4(3)$ & & 1 \\
\hline
\end{tabular}

Data are presented as mean \pm SD or number (fibroelastic deficiency; NYHA, New York Heart Association; $\mathrm{LV}$, left ventricle; LA, left atrium; MR, mitral regurgitation; TR, tricuspid regurgitation.

Table 2. Surgical procedure.

\begin{tabular}{|c|c|c|c|c|}
\hline & All $(\mathrm{n}=172)$ & No event group $(\mathrm{n}=157)$ & AA event group $(\mathrm{n}=15)$ & $\mathrm{P}$ \\
\hline Left atrial approach, n (\%) & $119(74)$ & $109(75)$ & $10(67)$ & 0.54 \\
\hline New chordal replacement, n $(\%)$ & $148(86)$ & $134(85)$ & $14(93)$ & 0.7 \\
\hline Resection, n (\%) & $19(11)$ & $18(11)$ & $1(7)$ & 1 \\
\hline Leaflet augmentation, n (\%) & $3(2)$ & $3(2)$ & $0(0)$ & 1 \\
\hline Tricuspid valve annuloplasty & $23(13)$ & $21(13)$ & $2(13)$ & 1 \\
\hline Coronary artery bypass graft, $\mathrm{n}(\%)$ & $18(11)$ & $14(9)$ & $4(27)$ & 0.055 \\
\hline Full ring, $\mathrm{n}(\%)$ & $170(99)$ & $156(99)$ & $14(93)$ & 0.17 \\
\hline Ring size, $\mathrm{mm}$ & $28.1 \pm 2.3$ & $28.3 \pm 2.3$ & $28.5 \pm 1.9$ & 0.75 \\
\hline Prosthetic orifice area, $\mathrm{mm}^{2}$ & $395 \pm 76$ & $395 \pm 77$ & $404 \pm 61$ & 0.67 \\
\hline Index prosthetic orifice area, $\mathrm{mm}^{2} / \mathrm{m}^{2}$ & $247 \pm 48$ & $247 \pm 49$ & $247 \pm 29$ & 0.99 \\
\hline Cardiopulmonary bypass time, min & $168 \pm 53$ & $165 \pm 50$ & $195 \pm 69$ & 0.035 \\
\hline Second cardiopulmonary bypass, n (\%) & $21(13)$ & $20(13)$ & $1(7)$ & 0.7 \\
\hline
\end{tabular}

Data are presented as mean $\pm \mathrm{SD}$ or number (

Table 3. Postoperative echocardiography.

\begin{tabular}{lllll}
\hline & All $(\mathrm{n}=172)$ & No event group $(\mathrm{n}=157)$ & AA event group $(\mathrm{n}=15)$ & $\mathrm{P}$ \\
\hline LV mass index, g/m & $102 \pm 33$ & $101 \pm 33$ & $117 \pm 30$ & 0.12 \\
LV end-diastolic diameter, mm & $45.9 \pm 7.9$ & $46.1 \pm 8.1$ & $44.8 \pm 5.1$ & 0.56 \\
LV end-systolic diameter, mm & $34.0 \pm 16.5$ & $34.3 \pm 17.2$ & $30.7 \pm 4.0$ & 0.41 \\
LV ejection fraction, \% & $55.5 \pm 10.7$ & $55.4 \pm 11.0$ & $56.9 \pm 7.0$ & 0.63 \\
LA diameter, mm & $36.6 \pm 6.2$ & $36.3 \pm 6.1$ & $39.1 \pm 7.6$ & 0.2 \\
Residual MR >grade2, $\mathrm{n}(\%)$ & $2(1)$ & $1(1)$ & $3.4 \pm 0.7$ & 0.17 \\
Transmitral mean pressure gradient, $\mathrm{mmHg}$ & $3.1 \pm 1.2$ & $3.0 \pm 1.2$ & $20.9 \pm 7.9$ & 0.23 \\
TR peak pressure gradient, mmHg & $20.4 \pm 6.2$ & $20.4 \pm 6.1$ & $119 \pm 6$ & 0.83 \\
Aorto-mitral angle, degree & $125 \pm 10$ & $125 \pm 10$ & & 0.00 \\
\hline
\end{tabular}

Data are presented as mean $\pm \mathrm{SD}$ or number (ventricle; LA, left atrium; NYHA, New York Heart Association; 
MR, mitral regurgitation; TR, tricuspid regurgitation.

Table 4. Prediction for new onset atrial arrhythmia.

\begin{tabular}{lllll}
\hline & Univariate & Univariate & Multivariate & Multivariate \\
\hline & OR $(95 \% \mathrm{CI})$ & $\mathrm{P}$ & $\mathrm{OR}(95 \% \mathrm{CI})$ & $\mathrm{P}$ \\
Age & $1.01(0.97-1.06)$ & 0.63 & - & - \\
Hypertension & $1.35(0.49-3.75)$ & 0.56 & - & - \\
Dyslipidemia & $1.00(0.31-3.22)$ & 1 & - & - \\
Diabetes mellitus & $1.93(0.52-7.11)$ & 0.32 & - & - \\
LV ejection fraction at the pre-operative TTE & $1.01(0.94-1.08)$ & 0.86 & - & - \\
LA diameter at the pre-operative TTE & $0.98(0.90-1.08)$ & 0.73 & - & - \\
Aorto-mitral angle at the pre-operative TTE & $0.98(0.93-1.03)$ & 0.39 & - & - \\
Anterior mitral leaflet prolapse & $2.57(0.91-7.29)$ & 0.08 & - & - \\
Posterior mitral leaflet prolapse & $0.43(0.16-1.20)$ & 0.11 & - & - \\
New chordal replacement & $0.96(0.12-7.52)$ & 0.97 & - & - \\
Leaflet resection & $0.87(0.11-6.77)$ & 0.9 & - & - \\
Coronary bypass graft & $2.64(0.82-8.56)$ & 0.1 & - & - \\
CPB time per 10min & $1.10(1.00-1.20)$ & 0.047 & $1.12(1.03-1.21)$ & 0.008 \\
LV ejection fraction at the post-operative TTE & $1.01(0.96-1.07)$ & 0.69 & - & - \\
LA diameter at the post-operative TTE & $1.08(0.97-1.21)$ & 0.17 & - & - \\
Residual MR (greater than moderate) & $1.68(0.19-14.6)$ & 0.64 & - & - \\
Mean TMPG at the post-operative TTE & $1.55(0.99-2.43)$ & 0.054 & - & - \\
Aorto-mitral angle at post-operative TTE & $0.93(0.87-0.99)$ & 0.025 & $0.92(0.86-0.98)$ & 0.014 \\
\hline
\end{tabular}

CI, confidence interval; CPB, cardiopulmonary bypass; LA, left atrial; LV, left ventricle; MR, mitral regurgitation; OR, odds ratio; TMPG, transmitral pressure gradient; TTE, transthoracic echocardiography.

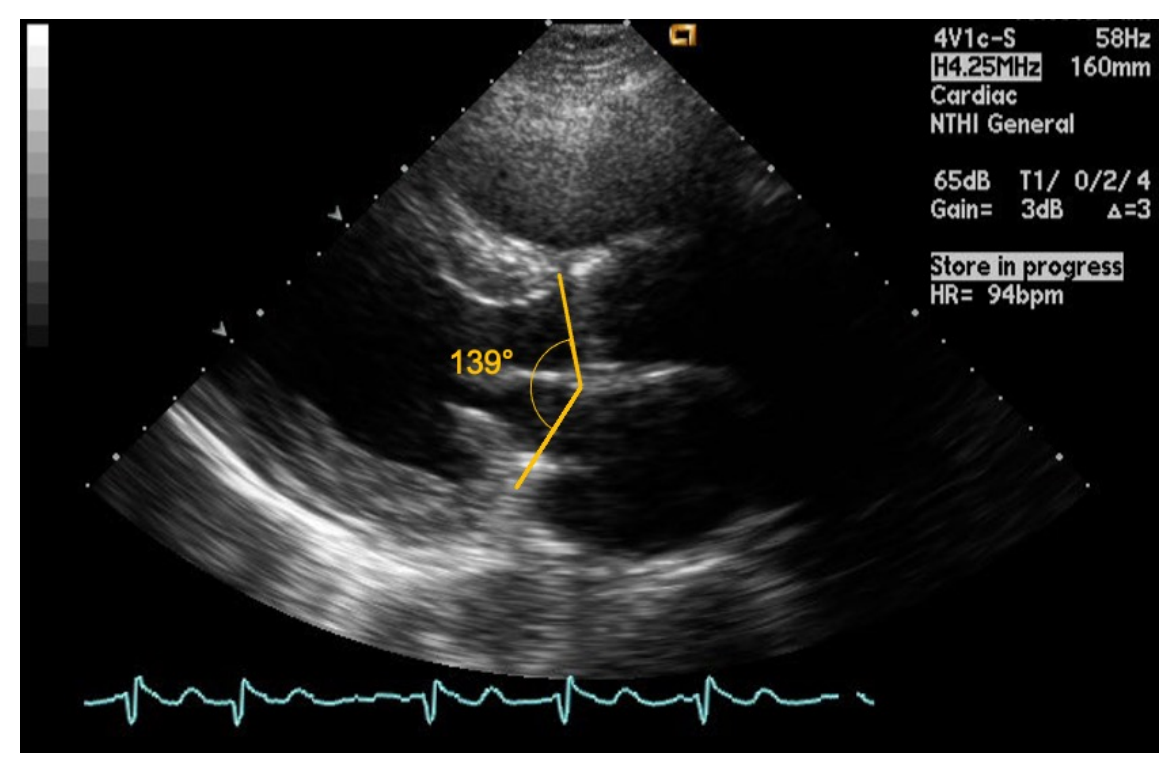




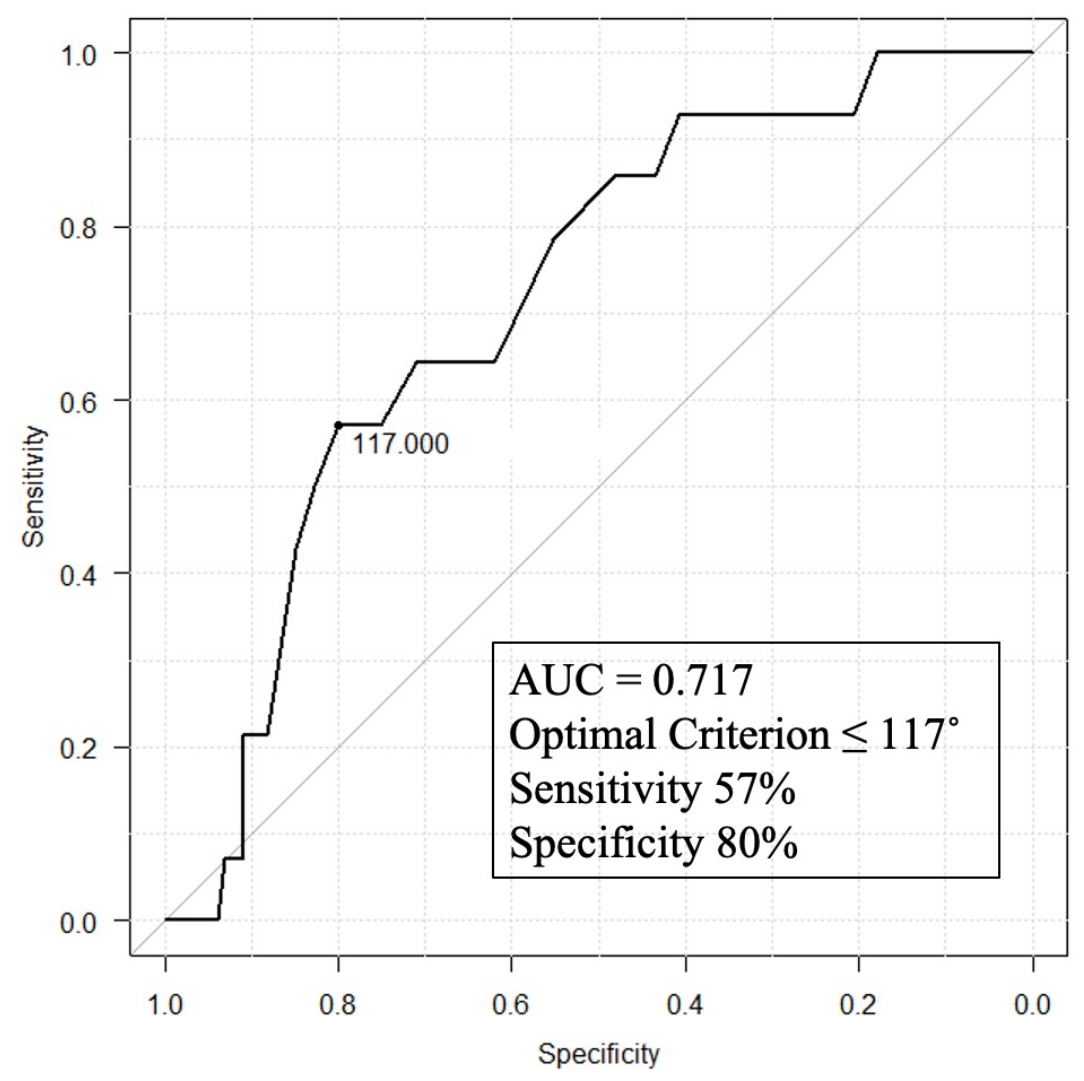



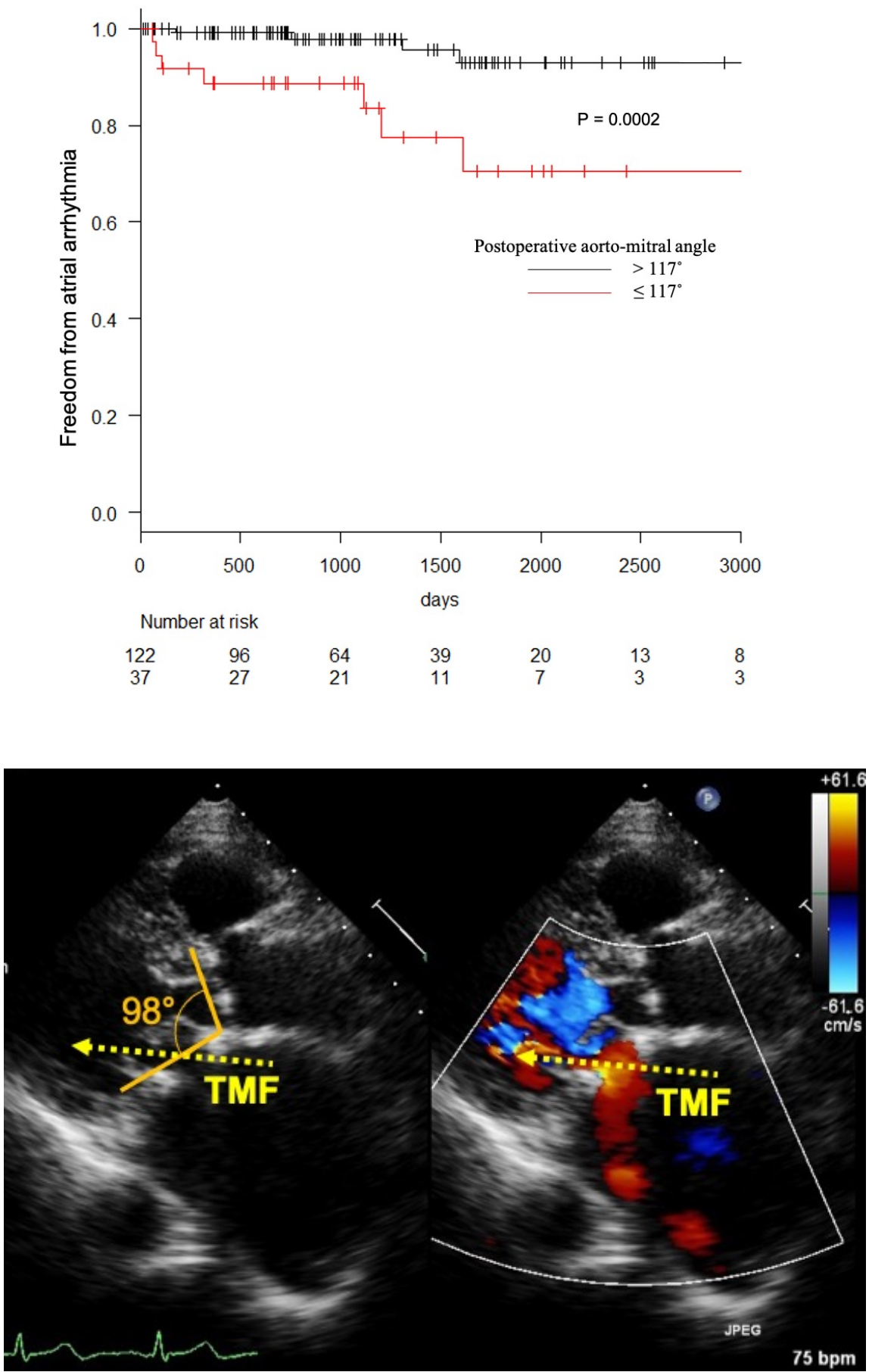